\title{
Standards-Compatible Compression for Automated Image Recognition in Sensor Networks and Surveillance Systems
}

\author{
Mo Chen, Mark L. Fowler ${ }^{\dagger}$, Shuqun Zhang \\ Department of Electrical and Computer Engineering \\ State University of New York at Binghamton \\ Binghamton, NY 13902
}

\begin{abstract}
We show that the standard image compression algorithms are not suitable for compressing images in correlation pattern recognition since they aim at retaining image fidelity in terms of perceptual quality rather than preserving spectrally significant information for pattern recognition. New compression algorithms for pattern recognition are therefore developed, which are based on the modification of the standard compression algorithms to achieve higher compression ratio and simultaneously to enhance pattern recognition performance. This is done by emphasizing middle and high frequency components and discarding low frequency components according to a new developed distortion measure for compression. The operations of denoising, edge enhancement and compression can be integrated in the encoding process in the proposed compression algorithms. Simulation results show the effectiveness of the proposed compression algorithms.
\end{abstract}

Keywords: data compression, distortion measure, distortion criteria, sensor network, surveillance system

\section{INTRODUCTION}

The use of correlation for pattern recognition has been extensively investigated due to its several advantages compared with other pattern recognition methods, which may include easy fast optical implementation, shiftinvariance, relative sensor independence and no need of target segmentation. The classical matched spatial filter $(\mathrm{MSF})^{1}$ is often not adequate for practical pattern recognition since its performance degrades rapidly when the reference object is distorted. Therefore, many improvements in correlation filters in the frequency domain ${ }^{2-5}$, and pre- $^{6-8}$ and post ${ }^{9-11}$ processing techniques in the spatial domain have been explored. Most of them involve the design of filters tolerable to various distortions such as noise, scale change, rotation and illumination. Pre-processing (such as denoising, edge enhancement and binarization) of the input image before correlation and post-processing of the correlation output also has been demonstrated to be effective in improving the performance of correlation-based pattern recognition systems.

Recently, there is some interest in investigating the integration of image compression and pattern recognition, and the impact of compression on pattern recognition ${ }^{12,13}$. This is partially motivated by the need to compress images in time-critical and energy-critical distributed image processing applications such as sensor networks ${ }^{14}$ and other surveillance systems, where the images must be transferred from one node to the other before the target recognition task can be completed as shown in Figure 1. Due to the low bandwidth of the data link and large throughput of image, lossy image compression method must be employed in order to accomplish the image transfer within the time requirement. In addition, data compression has been advocated as a means to achieve energy efficiency in sensor networks ${ }^{14}$. A common approach is to use standard image compression such as JPEG or a wavelet-based algorithm to compress the image. Walls and Mahalanobis ${ }^{12}$ have studied the impact of the waveletbased compression on the performance of automatic target recognition system that uses the maximum average correlation height filter (MACH) and the distance classifier correlation filter (DCCF). It was shown that these two correlation filters can recognize patterns in IR and SAR imagery at high compression ratios. Typically, the compression image is required to be uncompressed first before the recognition algorithm can be applied. In order to avoid the explicit image reconstruction, Mahalanobis and Daniell ${ }^{13}$ suggested integrating the recognition and compression tasks by merging the inverse wavelet transform and correlation filtering operations.

\footnotetext{
† Correspondence: mfowler@binghamton.edu
} 
An important observation is that standard image compression algorithms might not be able to achieve the highest compression ratio and the best performance when applied to compress images for correlation pattern recognition (CPR). This is because standard image compression algorithms aim at retaining image fidelity in terms of perceptual quality and mean square error (MSE) rather than preserving spectrally significant information for pattern recognition. Standard image compression algorithms are based on the fact that human eyes are typically less sensitive to high frequency oscillatory patterns than to low frequency variations. Therefore the components of high frequencies are usually considered unimportant and deleted in the existing image compression algorithm. However, pattern recognition does not require pixel level reconstruction of visual information and, as seen from our theoretical discussion in Section 3, in CPR the high frequency components are more important than the low frequencies. But since the low frequency coefficients tend to be much larger than the high frequency coefficients, standard compression algorithms will allocate a large number of bits for the low frequency coefficients and will therefore be ill-suited to CPR applications. Thus, in CPR applications a much higher compression ratio can be achieved if we discard the low frequency components. The elimination of low frequencies won't degrade and might even enhance the recognition performance since it corresponds to edge enhancement operation.

Based on the above observations, in this paper we show that standard image compression algorithms are not suitable for pattern recognition. Perhaps the simplest strategy in compressing images for recognition purpose is to use a mask to preserve high frequency components only. However, this will reduce the noise tolerance capability of the pattern recognition system. To keep the noise tolerance and discrimination capability, only the middle frequency components should be retained and coded during compression. A major concern here is how to preserve coefficients. This requires the development of a CPR-driven distortion measure for compressing images to use in place of distortion measures used in standard compression techniques. Fowler ${ }^{15-17}$ proposed a non-MSE measure for one-dimensional signal compression that is well-suited for emitter location systems. In Section 3 of this paper it is extended to two-dimensional image compression and shown to be applicable to the CPR compression problem. Based on this criterion, we modify standard compression algorithms so they can be used for CPR to achieve higher compression ratio and enhanced recognition performance. The desire to simply modify standard compression algorithms springs from the great advantage this brings to system developers who can then have access to highperformance, CPR-specific algorithms through simple modification of readily-available software that implements standard algorithms. The proposed algorithms combine the operations of denoising, edge enhancement and compression in the encoding process, while leaving the decoding intact. They are general for any correlation filters.

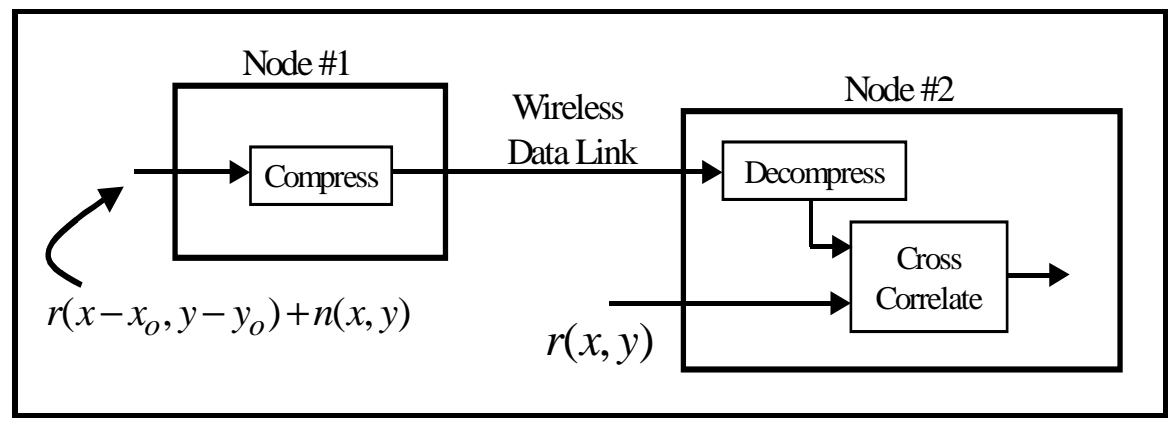

Figure 1: Sensor network application using cross correlation for image recognition.

\section{STANDARD IMAGE COMPRESSION ALGORITHMS}

JPEG and wavelet-based compression are the two most widely used lossy image compression algorithms. We briefly review their principle and point out that they are not optimal compression algorithms for CPR.

JPEG compression is based on the 2-D discrete cosine transform (DCT) that has high energy-compactness capability and a fast algorithm. An image to be compressed is first divided into $8 \times 8$ blocks of pixels, which are then transformed to the frequency domain using a DCT. The 2-D DCT transform puts low frequency coefficients into the upper left corner of the block of DCT coefficients and high frequency coefficients into the lower right corner. Many blocks will have significant coefficients only at low frequencies and thus in the upper left of each block. JPEG is based on the fact that our visual perception is typically less sensitive to high frequency than to low frequency variations. To minimize the visual degradation of the coded images, JPEG performs a quantization to keep low 
frequency coefficients and discard high frequency coefficients by dividing the DCT coefficients with a quantization matrix and then rounding the results ${ }^{18}$; a typical quantization matrix is:

$$
Q_{\text {JPEG }}=\left[\begin{array}{cccccccc}
16 & 11 & 10 & 16 & 24 & 40 & 51 & 61 \\
12 & 12 & 14 & 19 & 26 & 58 & 60 & 55 \\
14 & 13 & 16 & 24 & 40 & 57 & 69 & 56 \\
14 & 17 & 22 & 29 & 51 & 87 & 80 & 62 \\
18 & 22 & 37 & 56 & 68 & 109 & 103 & 77 \\
24 & 35 & 55 & 64 & 81 & 104 & 113 & 92 \\
49 & 64 & 78 & 87 & 103 & 121 & 120 & 101 \\
72 & 92 & 95 & 98 & 112 & 100 & 103 & 99
\end{array}\right] .
$$

The quantization weights are small in the upper left (low frequencies) and large in the lower right (high frequencies). The result of the division is that many high frequency coefficients become zero after the rounding and the low frequency coefficients undergo only minor adjustment. After quantization JPEG converts each quantized block into a 64-element vector using the zig-zag order and codes it using run-length-encoding followed by lossless entropy coding to get further compression ${ }^{18}$. For decompression of a JPEG file, the reverse processes are performed to reconstruct the image from compressed stream, where knowledge of the quantization matrix used by the encoder is needed - if the standard matrix has been used no additional side information is needed.

Wavelet-based image compression has been demonstrated to provide better compression performance than the DCT-based JPEG method at high levels of compression. The embedded zerotree wavelet (EZW) algorithm is one well-known wavelet-based compression algorithm ${ }^{19}$ for which software implementations are available. The wavelet transform decomposes the input image into a set of subbands of varying resolutions - these subbands represent different frequency bands and the coefficients within a given subband span the spatial range of the image. EZW creates so-called zerotrees such that each zero-tree contains coefficients that represent the same spatial region but span multiple subbands. The zerotree is an efficient method to represent the wavelet structure and to quantize wavelet coefficients by discarding the coefficients of the same orientation and same spatial location at finer scales. Arithmetic coding is used to entropy code the quantized coefficients. Due to the multi-resolution nature of wavelet decomposition, higher compression ratio can be achieved and more high frequency components can be saved. Despite its differences from JPEG, EZW nonetheless is based on the same principle of discarding the high frequency coefficients.

In summary, the standard compression algorithms were developed for visualization purpose so they always preserve low frequency and discard high frequency coefficients. When applied to compress images for CPR, they are not optimal in terms of the achievable compression ratio and recognition performance because for CPR high frequencies play a much more important role in discrimination than low frequencies. Edge enhancement or deleting low frequencies has been shown to be very effective in improving CPR system's discrimination capability. Therefore, if we throw away low frequency components during compression, it will have several benefits. First, it can enhance the correlator's discrimination performance as stated earlier. Second, it can integrate edge enhancement and denoising into the compression process and thus reduces the computational complexity. Third, and the most important one, it can increase the compression ratio significantly and reduce the compression algorithm complexity as well. This is because the values of low frequency coefficients are much larger than high frequency ones, and we only need to encode those small middle to high frequency coefficients.

Since in sensor network applications the input scene may contain noise, it is not appropriate to keep only those extremely high frequency components. Generally a correlation filter should be bandpass in nature to balance the noise tolerance and discrimination capability. Therefore, we should retain middle frequency components instead of extremely high frequencies during compression. Thus we need to have a distortion criterion for guiding the design of new compression algorithms for CPR. The distortion measures used in standard compression methods are obviously not suitable here. We will derive a distortion measure for CPR in the next section. 


\section{A DISTORTION CRITERION FOR IMAGE COMPRESSION IN CPR}

Let $r(x, y)$ denote the target image and $s(x, y)$ be the observed input scene that contains the reference image $r(x, y)$ and additive input noise $n(x, y)$. When the target is located at unknown coordinates $\left(x_{0}, y_{0}\right)$, the input scene can be written as

$$
s(x, y)=r\left(x-x_{0}, y-y_{0}\right)+n(x, y)
$$

The noise $n(x, y)$ is assumed to be zero-mean additive Gaussian noise. Let $h(x, y)$ be the correlation filter being used. The resulting correlation output $c(x, y)$ is given by

$$
c(x, y)=\iint s(m, n) h(m+x, n+y) d m d n .
$$

In particular, $h(x, y)$ is the MSF when $h(x, y)=r(x, y)$ and is optimal in sense of producing the maximum output signal-to-noise ratio (SNR).

Correlators must be able not only to identify the objects in an input image but also to locate the desired target in the input scene. Ideally, $c(x, y)$ should have a large peak located at $(x, y)=\left(x_{0}, y_{0}\right)$ and small values elsewhere. However, the noise in the input scene can obscure the peak and/or shift it from its nominal position. The peak's coordinates denoted by $\left(\hat{x}_{0}, \hat{y}_{0}\right)$ can by estimated using maximum a posteriori (MAP) estimate,

$$
\left.\left(\hat{x}_{0}, \hat{y}_{0}\right)=\underset{\left(x_{0}, y_{0}\right)}{\arg \max } \iiint s(x, y) r\left(x-x_{0}, y-y_{0}\right) d x d y+N_{0} \ln \left(p\left(x_{0}, y_{0}\right)\right)\right\}
$$

where $N_{0}$ is the noise spectral height and $p\left(x_{0}, y_{0}\right)$ is the coordinate a priori distribution density. It can be proven ${ }^{20}$ that the Cramer Rao Lower Bound on the variances of the location errors in $x$ - and $y$-directions are given by

$$
\begin{gathered}
\sigma_{x}^{2} \geq \frac{c_{x} \times N_{0}}{4 \pi^{2} \iint u^{2}|R(u, v)|^{2} d u d v}, \\
\sigma_{y}^{2} \geq \frac{c_{y} \times N_{0}}{4 \pi^{2} \iint v^{2}|R(u, v)|^{2} d u d v},
\end{gathered}
$$

where $R(u, v)$ is the Fourier transform of $r(x, y)$, and $u$ and $v$ are the frequencies along the $x$-axis and $y$-axis. $c_{x}$ and $c_{y}$ are some constants. From (4) and (5), we can see that the accuracy of locating the reference image in the received image using the MSF is governed by

$$
\begin{aligned}
& F_{x}^{2}=\sum_{v=0}^{M-1} \sum_{u=0}^{N-1} u^{2}|R(u, v)|^{2}, \\
& F_{y}^{2}=\sum_{v=0}^{M-1} \sum_{u=0}^{N-1} v^{2}|R(u, v)|^{2},
\end{aligned}
$$

in a discrete format, where $M \times N$ is the size of $r(x, y)$. Large $F_{x}^{2}$ and $F_{y}^{2}$ can result in lower variance of localization errors. It also can be seen from (6) and (7) that the high frequency coefficients in $R(u, v)$ are weighted more heavily than the low frequency coefficients. This implies that high frequency components play a more 
important role than low frequency components in CPR. So for compressing images for CPR, more weight should be given to the important high frequency components instead of to the low frequencies.

Since maximizing $F_{x}^{2}$ and $F_{y}^{2}$ can achieve improved location accuracy in pattern recognition, (6) and (7) can be used as a criteria for image compression in CPR. If we treat the $x$ and $y$ directions equally important, we can add $F_{x}^{2}$ and $F_{y}^{2}$ to form a single criterion as follows:

$$
F^{2}=F_{x}^{2}+F_{y}^{2}=\sum_{v=0}^{M-1} \sum_{u=0}^{N-1}\left(u^{2}+v^{2}\right)|R(u, v)|^{2} .
$$

\section{MODIFYING THE STANDARD COMPRESSION ALGORITHMS FOR CPR}

Our proposed compression algorithms are based on the modification of the standard compression algorithms. We only change their quantization step and leave others untouched. The advantage of this is that we are still able to use the standard decoders to decompress the images encoded by the proposed algorithms, which is desirable since it allows system engineers to easily and inexpensively apply our methods to practical systems. It should be noted that the methods we are proposing here are not necessarily the best at achieving optimization with respect to the criterion developed in Section 3, but rather are using that criterion as a guide to how to make standards-compatible modifications to JPEG and other compression methods. For more general approaches to application of our distortion measure to pattern recognition in the sensor network context, see Chen and Fowler ${ }^{14}$.

We modify the JPEG so it can be more suitable for CPR. Since the DCT is closely related to the Discrete Fourier Transform (DFT), Equation (8) can be replaced by the following for each of $8 \times 8$ DCT blocks

$$
D^{2}=\sum_{i=0}^{7} \sum_{j=0}^{7}\left(i^{2}+j^{2}\right) d c t^{2}(i, j)
$$

where $\operatorname{dct}(i, j)$ is the DCT coefficient at $(i, j)$ location. For the criterion obtained in Section 3, we know that high frequency DCT coefficients should be weighted and retained with refined quantization, whereas low frequency DCT coefficients should be deleted or quantized coarsely. This can be done by carefully selecting a quantization table. For simplicity, we rotate the JPEG's quantization matrix by $180^{\circ}$ and use it as our quantization matrix, which is shown as follows

$$
Q_{M O D}=\left[\begin{array}{cccccccc}
99 & 103 & 100 & 112 & 98 & 95 & 92 & 72 \\
101 & 120 & 121 & 103 & 87 & 78 & 64 & 49 \\
92 & 113 & 104 & 81 & 64 & 55 & 35 & 24 \\
77 & 103 & 109 & 68 & 56 & 37 & 22 & 18 \\
62 & 80 & 87 & 51 & 29 & 22 & 17 & 14 \\
56 & 69 & 57 & 40 & 24 & 16 & 13 & 14 \\
55 & 60 & 58 & 26 & 19 & 14 & 12 & 12 \\
61 & 51 & 40 & 24 & 16 & 10 & 11 & 16
\end{array}\right]
$$

this simple modification allows application of JPEG software with only minor modifications that are easily made. This rotated matrix can perform fine quantization for high frequency DCT coefficients and coarse quantization for low frequency DCT since quantization stepsize are large at the upper-left and small at the lower-right. Although this modification alone works toward the objective asserted in Section 3, it was found that further improvements were possible with additional modifications. Our modified JPEG method for CPR involves the following steps. After the DCT transform, each DCT coefficient is weighted by multiplying with $\sqrt{i^{2}+j^{2}}$, motivated by (9). The weighted coefficients are then quantized using the $180^{\circ}$ rotated quantization matrix. A mask can be used to delete those nonzero extremely high and low frequency coefficients. The obtained coefficients are finally entropy encoded. 
Since middle and high frequency components have much smaller magnitudes than low frequency components, a higher compression ratio than JPEG can be achieved.

We can also modify the wavelet-based image compression algorithms for CPR, while keeping the efficiency of wavelet-based coding. The wavelet-based algorithms are based on the multiresolution decomposition of the image to be compressed. For traditional visualization purpose, the LL (low frequency) band at the highest level is considered as most important, and the other bands containing edge information are classified as of less importance, with the degree of importance decreasing from the top of the pyramid to the bands at the bottom. As a result, a large number of bits are assigned to encode the LL band, and many coefficients at higher frequency subbands (filter resolution) are discarded. However, for pattern recognition, we know that edge information is considered more important. Accordingly, we suggest encoding the LH and HL bands. In this way, much higher compression ratio can be obtained while simultaneously enhancing the discrimination performance. Wavelet denoising methods ${ }^{21}$ also can be applied to the wavelet coefficients to reduce noise.

\section{SIMULATION RESULTS}

Computer simulations were performed to test the performance of the proposed algorithms. The input image used is an infrared image as shown in Figure 2 (a) (from the paper by Nguyen et al. ${ }^{22}$ ), and two reference images (small truck and big truck) are shown in Figure 2 (b) and (c). We first compare the performance of JPEG, EZW, and the proposed modified JPEG and EZW when the input image is noise free and then observe the performance in the presence of noise. In our proposed modified version of JPEG and EZW, lossless arithmetic coding after quantization is used. Additionally, in order to control the compression ratio (bit rate), we use the a scalar value $Q$ in our modified JPEG algorithm to adjust the degree of quantization, as following:

$$
q d c t(i, j)=\text { round }\left(\frac{d c t(i, j) \times \text { weight }(i, j)}{Q^{*} q u a n_{-} \text {matrix }(i, j)}\right) \times Q \times q u a n_{-} \text {matrix }(i, j),
$$

where $q \operatorname{dct}(i, j)$ is quantized DCT coefficient, quan_matrix $(i, j)$ is the proposed rotated $180^{\circ}$ quantization matrix and weight $(i, j)=\sqrt{i^{2}+j^{2}}$. Figure 3 shows the correlation surfaces (with mean value subtracted) after the input image is lossy compressed, reconstructed and cross-correlated with the reference using different compression algorithms. It is seen from Figure 3 (a) (b) (c) and (d) that the correlation distributions without compression, and with JPEG are almost the same (EZW is the same as JPEG). The correlation profiles are broad and there are a lot of sidelobes, making location/extraction of the refernce object's peak very difficult. Also in (a) and (c), the location of peaks are not at the true location of the small truck reference image because big truck has too much energy. Whereas, with the proposed modified JPEG and EZW compression, we obtain a much sharper correlation peak with a flat background, as shown in Figs, 2 (e)- (h). Most important, Figs 2 (e) and (g) give the correct location of the small truck reference. As far as compression ratio is concerned, the standard JPEG is around 30:1 and the modified JPEG is 73.5:1 with $Q$ equal to 3, and 270:1 for the modified EZW where only LH and HL components are coded in order to retain the efficiency of EZW scheme.

We increased the compression ratio of the modified JPEG method by adjusting the $\mathrm{Q}$ to values of $1,2,3,4,5$; under these conditions we were still able to recognize and correctly locate the reference image; the compression ratios achieved under these experiments are given in Table 1.

Table 1

\begin{tabular}{|c|l|l|l|l|l|}
\hline$Q$ & 1 & 2 & 3 & 4 & 5 \\
\hline CR & $11.5: 1$ & $33.5: 1$ & $73.5: 1$ & $135.5: 1$ & $209: 1$ \\
\hline
\end{tabular}

We can increase the compression ratio by eliminating more low frequencies components for modified JPEG compression. Moreover, because infrared images have most of zero coefficients in the very high frequency band, we only need to code the coefficients of middle frequency band. To simplify the coding, we can modified the weight 
from $\sqrt{i^{2}+j^{2}}$ to the $8 \times 8$ well-known Zig-Zag index and design the mask matrix to code only coefficients of middle frequencies from 16 to 39 , as follows

$$
\text { weight \& mask }(16: 39)=\left[\begin{array}{cccccccc}
0 & 1 & 5 & 6 & 14 & 15 & 27 & 28 \\
2 & 4 & 7 & 13 & 16 & 26 & 29 & 42 \\
3 & 8 & 12 & 17 & 25 & 30 & 41 & 43 \\
9 & 11 & 18 & 24 & 31 & 40 & 44 & 53 \\
10 & 19 & 23 & 32 & 39 & 45 & 52 & 54 \\
20 & 22 & 33 & 38 & 46 & 51 & 55 & 60 \\
21 & 34 & 37 & 47 & 50 & 56 & 59 & 61 \\
35 & 36 & 48 & 49 & 57 & 58 & 62 & 63
\end{array}\right] \times\left[\begin{array}{cccccccc}
0 & 0 & 0 & 0 & 0 & 0 & 1 & 1 \\
0 & 0 & 0 & 0 & 1 & 1 & 1 & 0 \\
0 & 0 & 0 & 1 & 1 & 1 & 0 & 0 \\
0 & 0 & 1 & 1 & 1 & 0 & 0 & 0 \\
0 & 1 & 1 & 1 & 1 & 0 & 0 & 0 \\
1 & 1 & 1 & 1 & 0 & 0 & 0 & 0 \\
1 & 1 & 1 & 0 & 0 & 0 & 0 & 0 \\
1 & 1 & 0 & 0 & 0 & 0 & 0 & 0
\end{array}\right]
$$

In this case, the decoder must know a priori the mask matrix and weight matrix as well, which is easily deced into the decoder. We call this the modified-mask JPEG algorithm. Table 2 shows the compression ratio obtained with this coding:

Table 2

\begin{tabular}{|c|c|c|c|c|c|c|c|c|c|}
\hline Q & 1 & 2 & 3 & 4 & 5 & 6 & 7 & 8 & 9 \\
\hline CR & 9:1 & $14: 1$ & $20: 1$ & 26.5:1 & $34: 1$ & 42:1 & $52: 1$ & $62: 1$ & $74: 1$ \\
\hline $\mathbf{Q}$ & 10 & 11 & 12 & 13 & 14 & 15 & 16 & 17 & 18 \\
\hline CR & $87: 1$ & 102:1 & 117:1 & $135: 1$ & 150:1 & 170:1 & 188:1 & 211:5 & 227:1 \\
\hline $\mathbf{Q}$ & 19 & 20 & 21 & 22 & 23 & 24 & 25 & 26 & 27 \\
\hline CR & 246:1 & 265:1 & 287:1 & 304:1 & $335: 1$ & 360:1 & 392:1 & 424:1 & 456:1 \\
\hline Q & 28 & 29 & 30 & 31 & 32 & 33 & 34 & 35 & \\
\hline CR & 481:1 & 510:1 & 541:1 & 577:1 & 645:1 & 697:1 & 742:1 & 820:1 & \\
\hline
\end{tabular}

Figures 4-6 show the reconstructed images and cross-correlation surface with the small truck reference using modified-mask JPEG when $Q$ is equal to 12,22 and 35 , respectively. We can go above $\mathrm{Q}=35$ to get even much higher compression ratio and still get the right location of the references. But in this case, we lost the edge of the tank and another vehicle in the input image. This can also been shown in Figure 6, where the edge of the two vehicles beside references disappear due to the coarse quantization.

Next, Gaussian noise with zero mean and standard deviation 50 was added to the input image. Using the modified JPEG algorithm, we can see from the Figure 7 that the accuracy of location (cross-correlation surface) does not degrade, but because the additive white Gaussian noise spread all of the coefficients, the compression ratio of our proposed algorithm will decrease. But images can be denoised before we perform our algorithm on the images. Genreral denoising is beyond the scope of this paper, but for the modified-mask JPEG the mask has some denoising capability because the coefficients of high frequencies contain more noise than image energy. Using the modified-mask JPEG with $\mathrm{Q}=15$, we varied the noise variance from 0 to 80 . Although the compression ratio decreases from 150:1 to 61:5, as shown in Table 3, we can still get benefits from our proposed algorithms. 
Table 3

\begin{tabular}{|l|l|l|l|l|l|l|l|l|l|}
\hline $\begin{array}{l}\text { AWGN } \\
\text { Variance }\end{array}$ & 0 & 10 & 20 & 30 & 40 & 50 & 60 & 70 & 80 \\
\hline CR & $150: 1$ & $138: 1$ & $122: 1$ & $106: 1$ & $94: 1$ & $84: 1$ & $76: 1$ & $67: 1$ & $61.5: 1$ \\
\hline
\end{tabular}

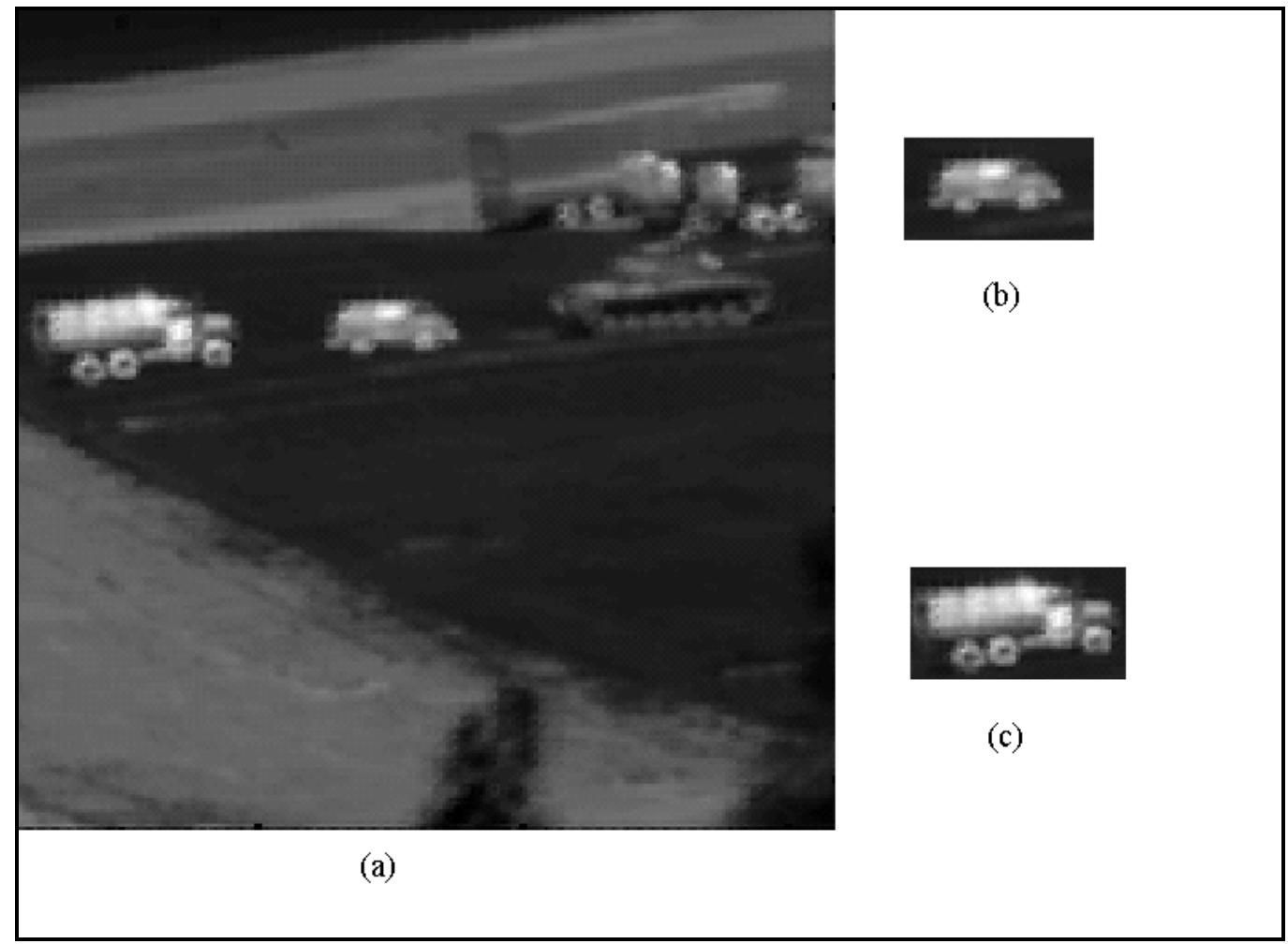

Figure 2: (a) Target image to be compressed (the image is from the paper by Nguyen et al. ${ }^{22}$ ); two reference images: (b) small truck and (c) big truck.

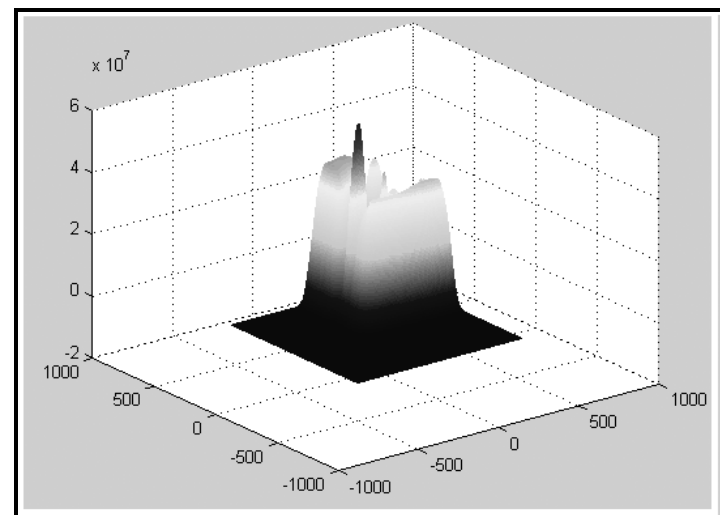

(a)

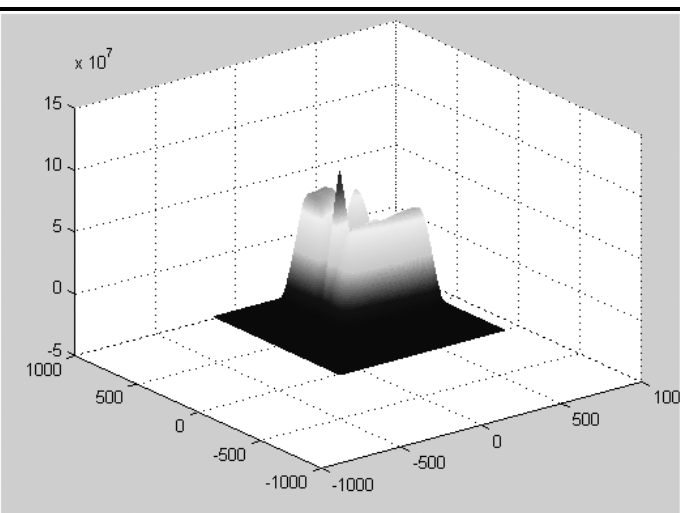

(b)

Figure 3: (a) Cross correlation surface between original input image and small truck reference image; (b) Cross correlation surface between original input image and big truck reference image. 


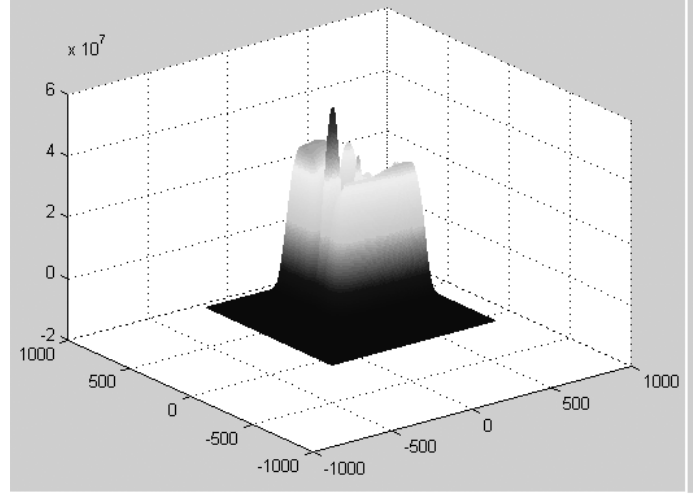

(c)

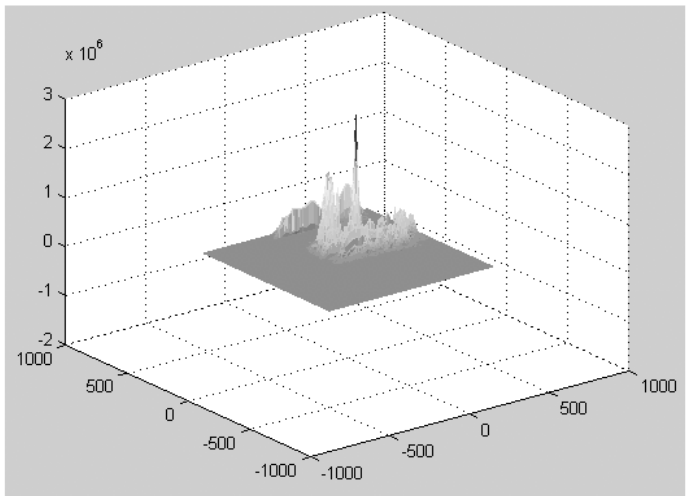

(e)

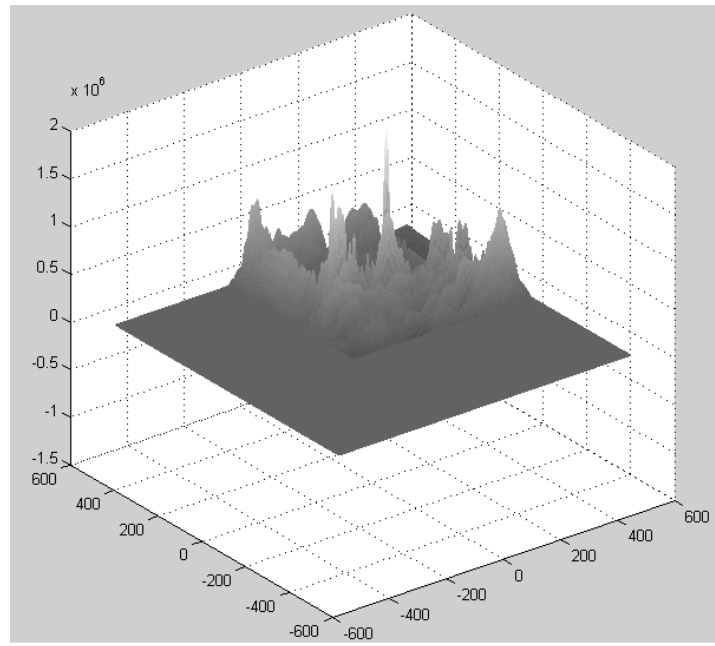

(g)

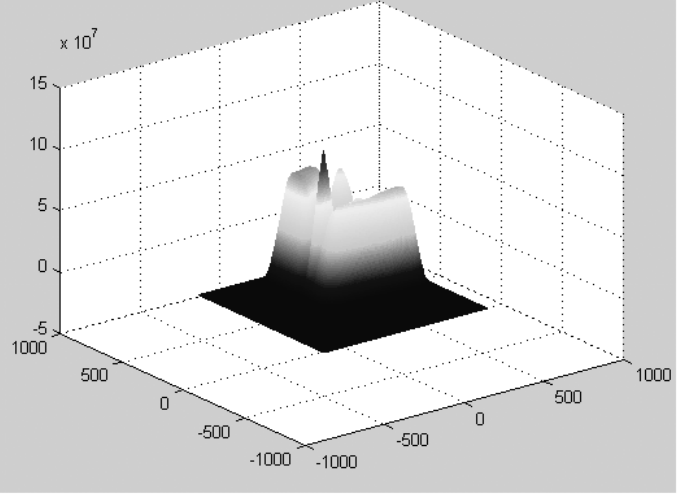

(d)

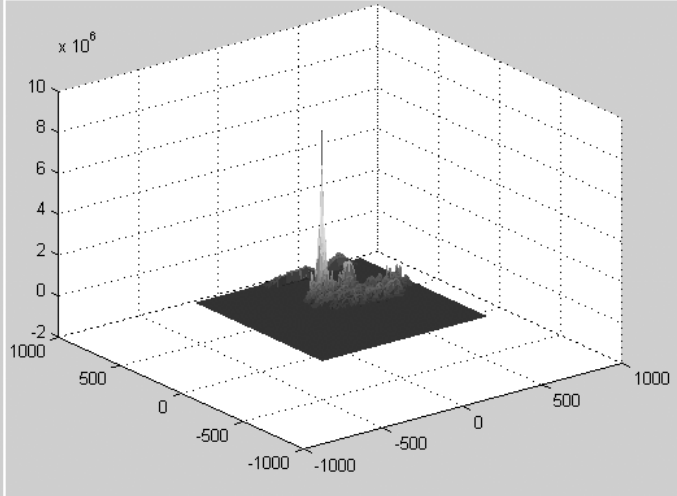

(f)

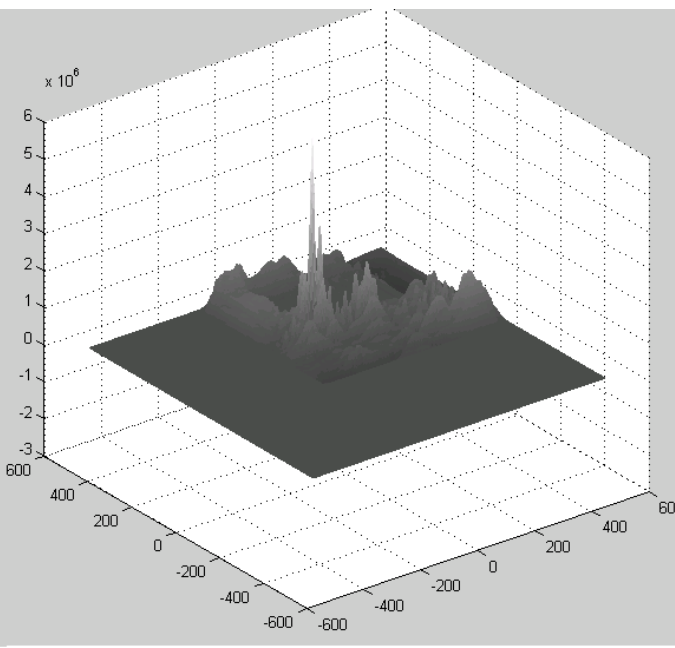

(h)

Figure 3 (cont.): Cross correlation surfaces between (c) standard JPEG processed input image and small truck reference image; (d) between standard JPEG processed input image and big truck reference image; (e) between our modified JPEG processed input image and small truck reference image; (e) between our modified JPEG processed input image and big truck reference image; (f) between our modified EZW processed input image and small truck reference image; (g) between our modified EZW processed input image and big truck reference image. 


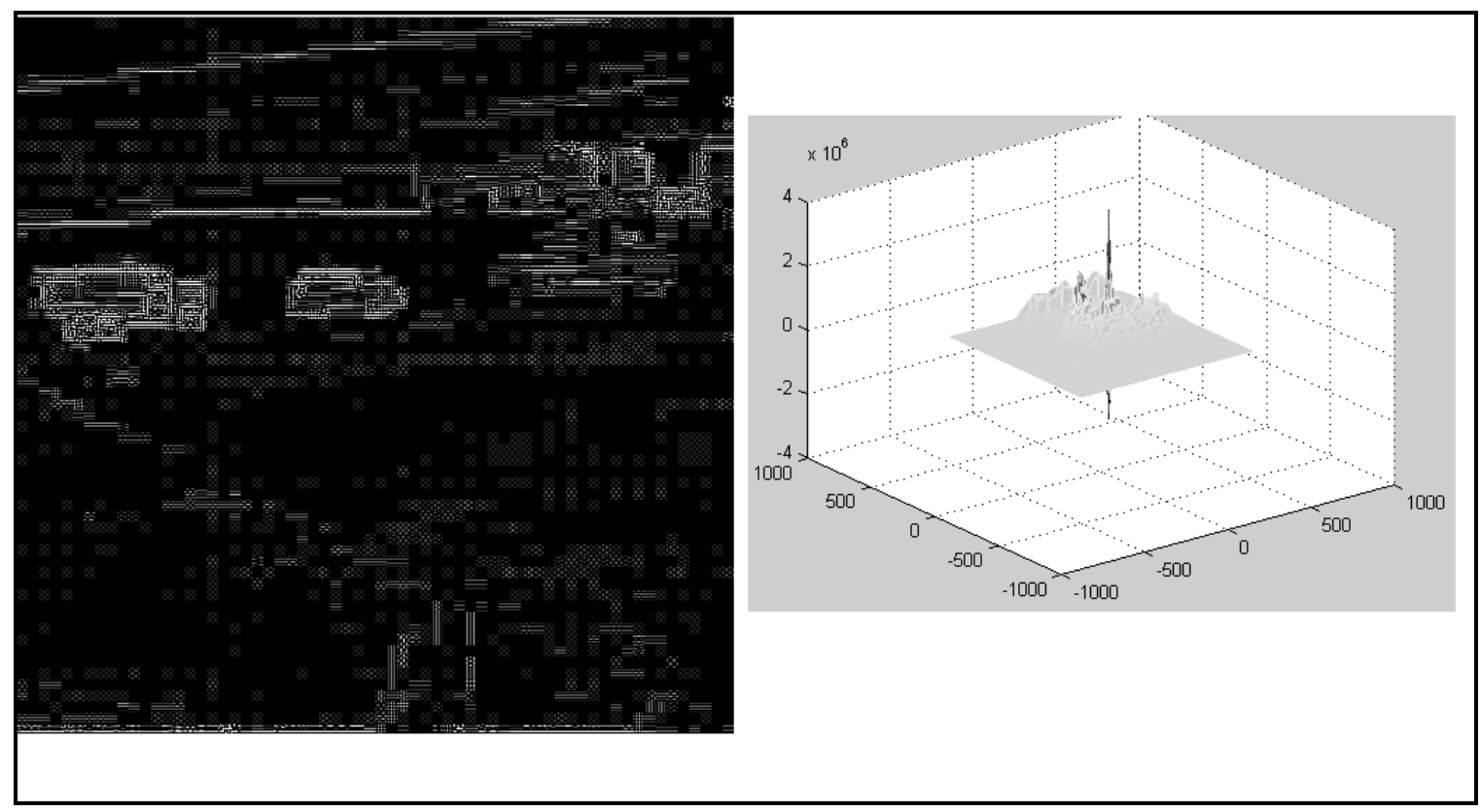

Figure 4: Reconstructed image and correlation with small truck reference using modified-mask JPEG with $\mathrm{Q}=12$.

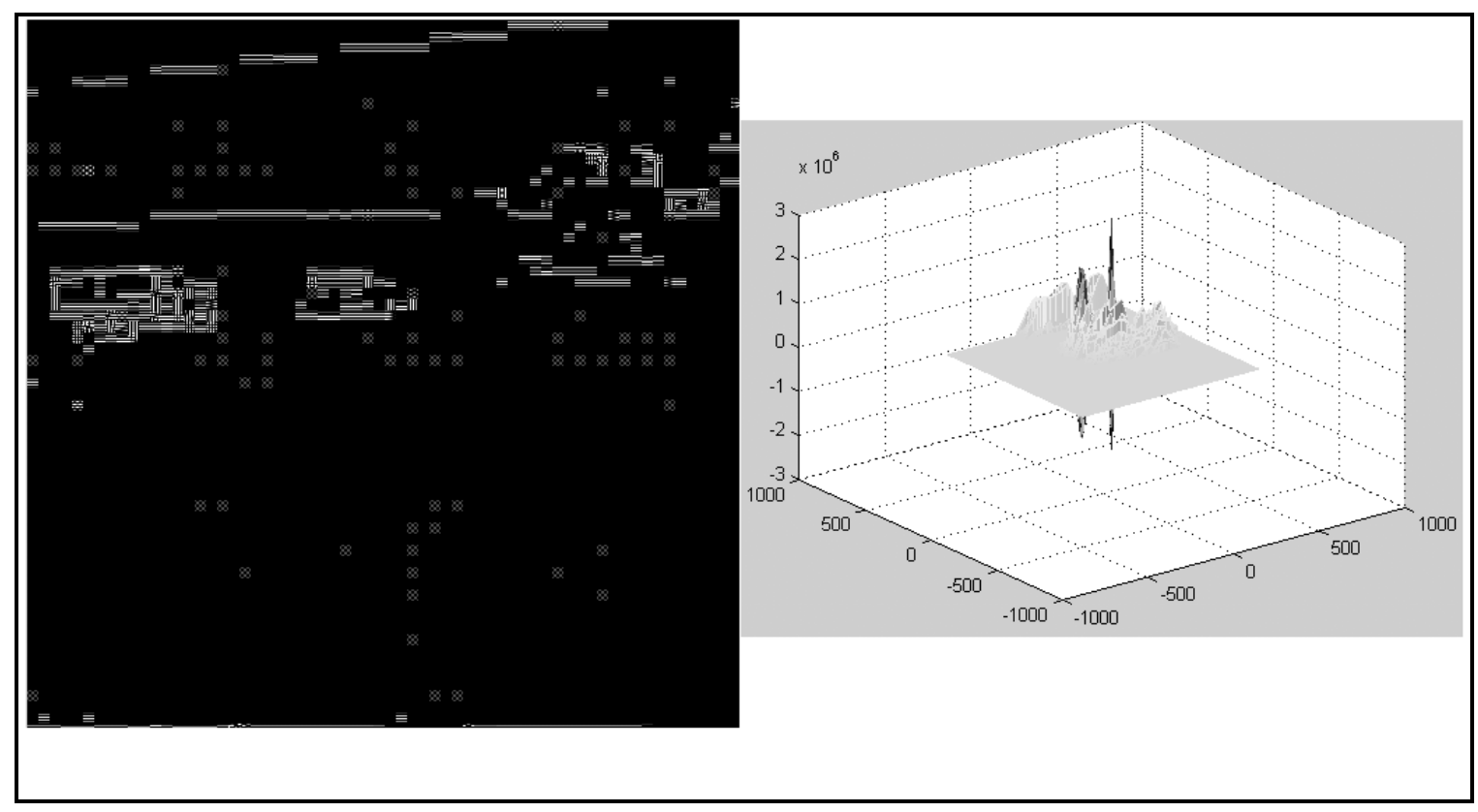

Figure 5: Reconstructed image and correlation with small truck reference using modified-mask JPEG with $\mathbf{Q}=\mathbf{2 2}$. 


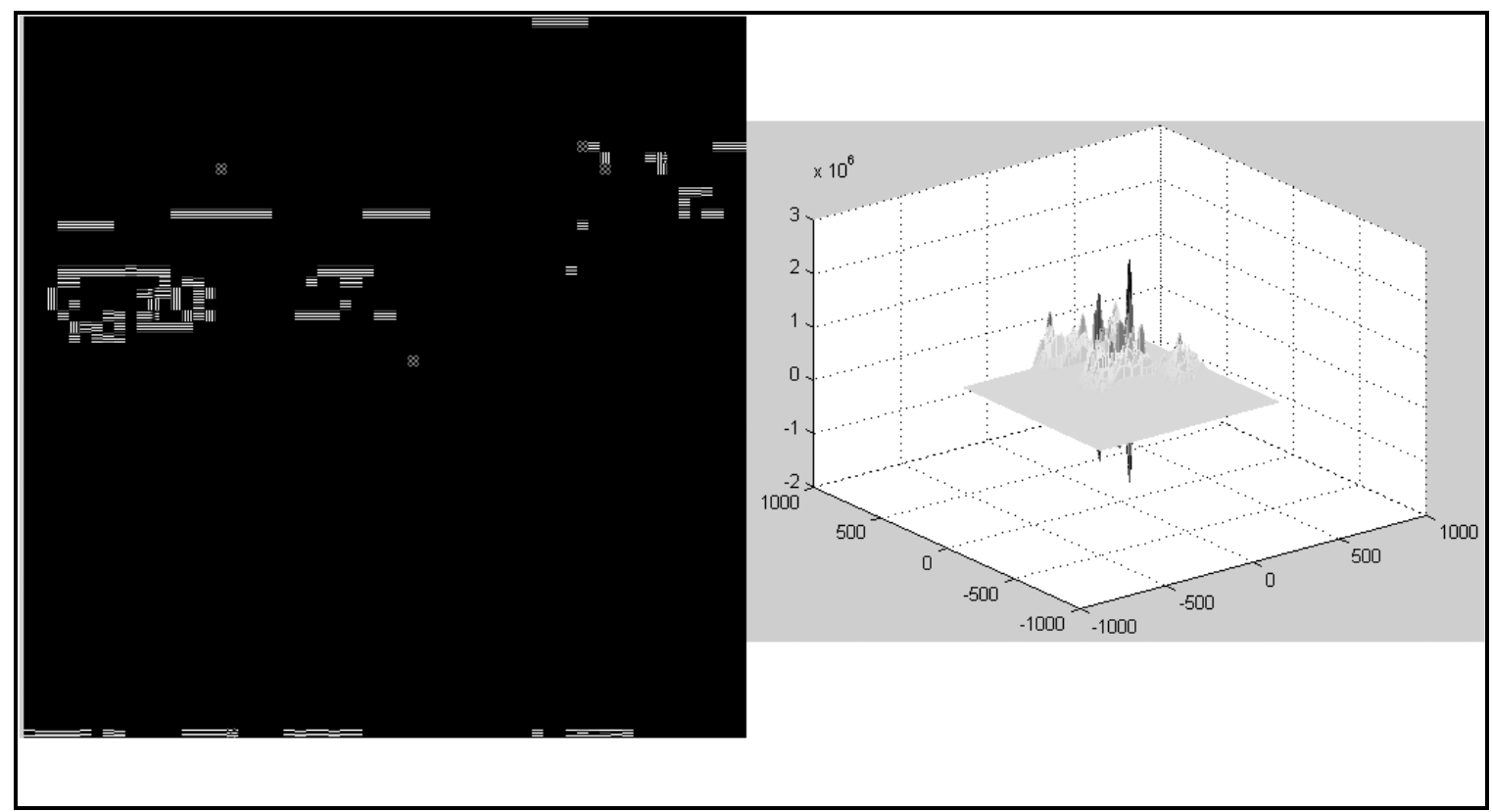

Figure 6: Reconstructed image and correlation with small truck reference using modified-mask JPEG with $\mathrm{Q}=35$.

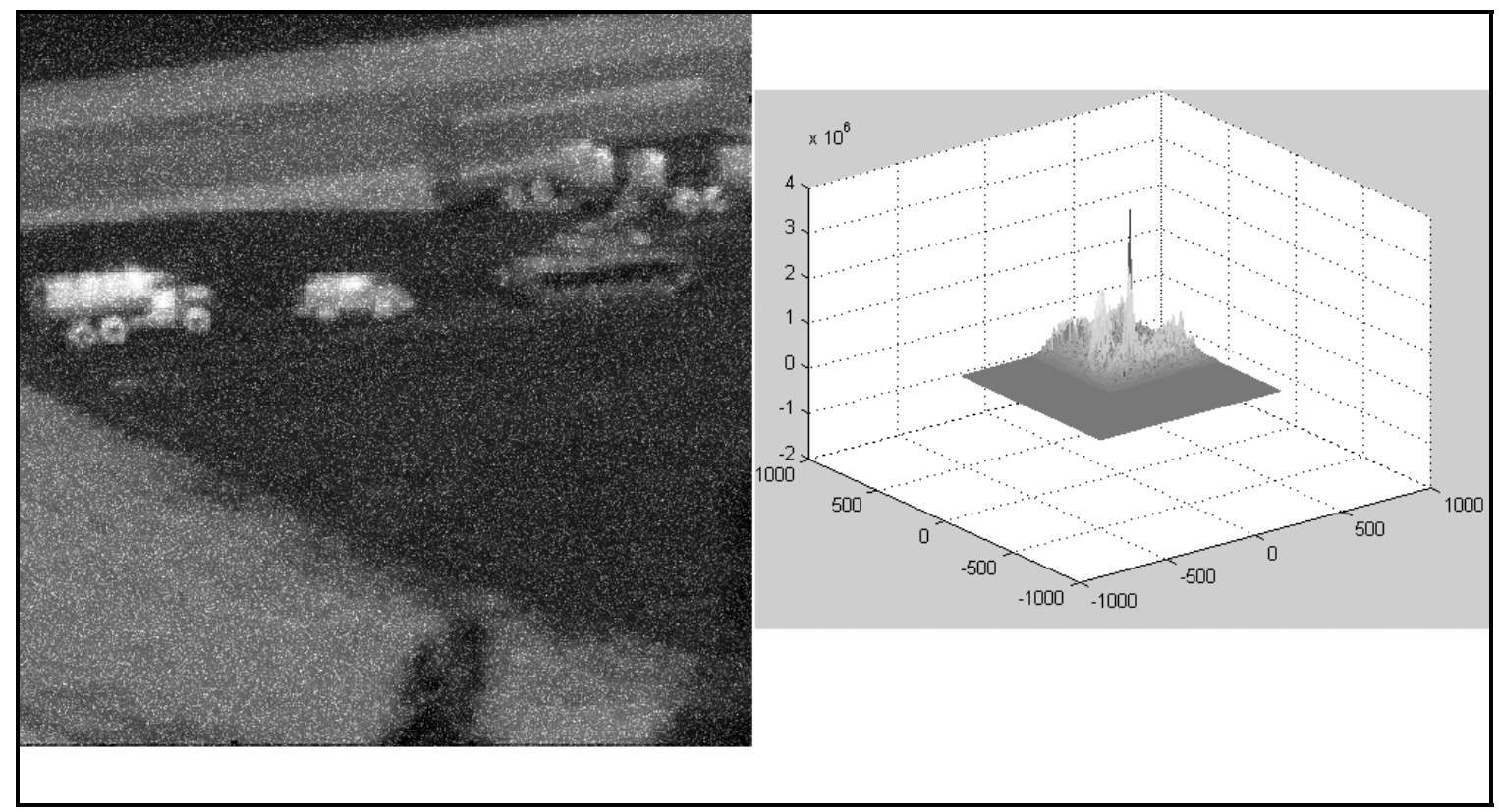

Figure 7: Noisy input image and correlation with small truck reference using modified-mask JPEG with $\mathrm{Q}=35$.

\section{CONCLUSIONS}

We have investigated image compression under the context of correlation pattern recognition. It has been pointed out that standard compression algorithms are good for visualization but not necessarily direct applicable for 
pattern recognition. In these methods, low frequency components are treated more important than high frequencies, but pattern recognition consider them oppositely. It is therefore possible to develop better compression techniques for pattern recognition based on the fact that pattern recognition does not require pixel level reconstruction of visual information but rather the preservation of spectrally significant information. We have discussed a new distortion measure for image compression and how to modify the standard compression algorithms to achieve both higher compression ratio and better recognition performance. The simulation results verify the performance of our proposed methods.

\section{REFERENCES}

1. A. VanderLugt, "Signal detection by complex spatial filtering," IEEE Trans. Inf. Theory, IT-10, 139-145 (1964)

2. B. V. K. Vijaya Kumar, "Tutorial survey of composite filter designs for optical correlators ," Appl. Opt., 31, 4773-4801 (1992)

3. B. V. K. Vijaya Kumar and A. Mahalanobis, "Recent advances in distortion-invariant correlation filter design," Proc. SPIE, 2490, 2-13 (1995)

4. A. Mahalanobis, B. V. K. Vijaya Kumar, S. Song, S. R. F. Sims, and J. F. Epperson, "Unconstrained correlation filters," Appl. Opt., 33, 3751-3759 (1994)

5. J. W. Fisher III and J. C. Principle, "Recent advances to nonlinear minimum average correlation energy filters," Opt. Eng, 36, 2697-2709 (1997)

6. L. S. Jamal-Aldin, R. C. D. Young, and C. R. Chatwin, "Application of nonlinearity to wavelet-transformed images to improve correlation filter performance," Appl. Opt., 36, 9212-9224 (1997)

7. W. C. Hasenplaugh and M. A. Neifeld, "Image binarization techniques for correlation-based pattern recognition," Opt. Eng., 38, 1907-1917 (1999)

8. L. S. Jamal-Aldin, R. C. D. Young, and C. R. Chatwin, "Synthetic discriminant function filter employing nonlinear space-domain preprocessing on bandpass filtered images," Appl. Opt., 37, 2051-2062 (1998)

9. M. Montes-Usategui, J. Campos, I. Juvells, and S. Vallmitjana, "Sidelobe elimination for generalized synthetic discriminant functions by a two-filter correlation and subsequent postprocessing of the intensity distribution," App. Opt., 33, 3050-3059 (1994)

10. A. Carnicer, S. Vallmitjana, I.Juvells, "Correlation postprocessing-based method for the detection of defocused images," Appl. Opt., 36, 4807-4811 (1997)

11. T. Wolf, B. Gutmann, H. Weber, J. Ferr-Borrull, S. Bosch, S. Vallmitjana, "Application of fuzzy-rule-based postprocessing to correlation methods in pattern recognition," Appl. Opt., 35, 6955-6963 (1996).

12. B. Walls and A. Mahalanobis, "Performance of the MACH filter and DCCF algorithms in presence of data compression," Proc. SPIE, 3718, 376-387 (1999)

13. A. Mahalanobis and C. Daniell, "Data compression and correlation filtering: A seamless approach to pattern recognition," Ch. 6 in Smart Imaging Systems, B. Javidi, editor, Bellingham, WA: SPIE Press, 2001.

14. M. Chen and M. L. Fowler, "The Importance of Data Compression for Energy Efficiency in Sensor Networks," Proceedings of Conference on Information Sciences and Systems, Johns Hopkins University, March 12-14, 2003.

15. M. L. Fowler, "Data compression for emitter location systems," Proceedings of Conference on Information Sciences and Systems, Princeton University, March 15-17, 2000, pp. WA 7b-14 - WA7b-19.

16. M. L. Fowler, "Decimation vs. quantization for data compression in TDOA systems, " Proc. SPIE, 4122, 56-67 (2000)

17. M. L. Fowler, "Non-MSE Wavelet-based Data compression for Emitter Location," Proc. SPIE, 4475, 13-22 (2001)

18. K. Sayood, Introduction to Data Compression, $2^{\text {nd }}$ Ed., San Francisco, CA: Morgan Kaufmann Publishers, 2000.

19. J. M. Shapiro, "Embedded image coding using zerotrees of wavelet coefficients," IEEE Trans. Signal Proc., SP-41, 3445-3462, (1993).

20. Steven M. Kay, Fundamentals of Statistical Signal Processing, Estimation Theory, Prentice-Hall, Inc. 1993

21. D. L. Donoho, "Denoising via soft thresholding ," IEEE Trans. Inf. Theory, 41, 613-627 (1995).

22. N. Nguyen, P. Milanfar, and G. Golub, "Efficient generalized cross-validation with applications to parametric image restoration and resolution enhancement," IEEE Trans. Image Proc., vol. 10, no. 9, 1299 - 130 (2001). 